\title{
Balanced-energy Sleep Scheduling Scheme for High Density Cluster-based Sensor Networks
}

\author{
Jing Deng*, Yunghsiang S. Han*†, Wendi B. Heinzelman ${ }^{\ddagger}$, and Pramod K. Varshney* \\ *Dept. of EECS, Syracuse University \\ Email: jdeng01, yshan, varshney@ecs.syr.edu \\ †Dept. of CSIE, National Chi Nan Univ., Taiwan, R.O.C. \\ Email: yshan@csie.ncnu.edu.tw \\ ${ }^{\ddagger}$ Dept. of ECE, University of Rochester \\ Email: wheinzel@ece.rochester.edu
}

\begin{abstract}
In order to conserve battery power in very dense sensor networks, some sensor nodes may be put into the sleep state while other sensor nodes remain active for the sensing and communication tasks. However, determining which of the sensor nodes should be put into the sleep state is non-trivial. As the goal of allowing nodes to sleep is to extend network lifetime, we propose and analyze a Balanced-energy Scheduling (BS) scheme in the context of cluster-based sensor networks. The BS scheme aims to evenly distribute the energy load of the sensing and communication tasks among all the nodes in the cluster, thereby extending the time until the cluster can no longer provide adequate sensing coverage. Two related sleep scheduling schemes, the Distance-based Scheduling (DS) scheme and the Randomized Scheduling (RS) scheme are also studied in terms of the coefficient of variation of their energy consumption. Analytical and simulation results are presented to evaluate the proposed BS scheme. It is shown that the BS scheme extends the cluster's overall network lifetime significantly while maintaining a similar sensing coverage compared with the DS and the RS schemes for sensor clusters.
\end{abstract}

\section{INTRODUCTION}

Recent technological advances have enabled the emergence of tiny, battery-powered sensors with limited on-board signal processing and wireless communication capabilities. Sensor networks may be deployed for a wide variety of applications [1]. A typical sensor network may contain thousands of small sensors, with the sensor density as high as 20 nodes $/ \mathrm{m}^{3}$. If these sensors are managed by the base station directly, communication overhead, management delay, and management complexity could make such a network less responsive and less energy efficient. Clustering has been proposed by researchers to group a number of sensors, usually within a geographic neighborhood, to form a cluster. Using a clustering approach, sensors can be managed locally by a cluster head, a node elected to manage the cluster and be responsible for communication between the cluster and the base station.

Clustering provides a convenient framework for resource management. It can support many important network features within a cluster, such as channel access for cluster members and power control, as well as between clusters, such as routing and code separation to avoid inter-cluster interference. Moreover, clustering distributes the management responsibility from the base station to the cluster heads. As pointed out by Varshney [2] and Heinzelman et al. [3], such distributed management provides a convenient framework for data fusion, local decision making and local control, and energy savings. A fixed or adaptive approach may be used for cluster maintenance. In a fixed maintenance scheme, cluster membership does not change over time. In an adaptive clustering scheme, however, nodes may change their associations with different clusters over time.

The sleeping technique has been used to conserve energy of battery powered sensors. Rotating active and inactive sensors in the cluster, some of which provide redundant data, is one way that sensors can be intelligently managed to extend network lifetime. Some researchers even suggest putting redundant sensor nodes into the network and allowing the extra sensors to sleep to extend the network lifetime [4]. This is made possible by the low cost of individual sensors.

When a sensor node is put into the sleep state, it completely shuts itself down, leaving only one extremely low power timer on to wake itself up at a later time. ${ }^{1}$ This leads to the following Sleep Scheduling Problem: How does the cluster head select which sensor nodes to put to sleep, without compromising the sensing coverage capabilities of the cluster?

In [6], we generalized and proposed two sleep scheduling schemes, termed the Randomized Scheduling (RS) scheme and the Distance-based Scheduling (DS) scheme. In the RS scheme, sensor nodes are randomly selected to go into the sleep state. In the DS scheme, the probability that a sensor node is selected to sleep depends on the distance it is located from the cluster head.

One possible drawback of the RS and the DS schemes is that the average energy consumptions of sensors with different distance to the cluster head might be different. In the RS scheme, all the sensor nodes in the cluster have the same sleep probability even though the sensor nodes on the border of the cluster may consume more energy than others. The DS scheme selects sensor nodes to sleep based on their distances from the cluster head, lowering the variation of energy consumptions by all sensor nodes. However, the coefficient of variation of

\footnotetext{
${ }^{1}$ Another approach is to use a low power wake-up circuit as in the WINS project, but a drawback of this approach is that it may suffer from the so-called "sleep deprivation torture attack" [5] by malicious nodes.
} 
sensor nodes' energy consumption could be relatively high. This is not desirable for sensor networks, as one of the design goals of the sleep scheduling scheme is to extend the network lifetime. If a certain fraction of the sensor nodes in the network consume much more energy than others, the batteries of these sensors die out quickly, creating holes (uncovered areas within the overall sensor network coverage area).

In this paper, we study the following Balanced-energy Sleep Scheduling Problem: How should a cluster head select nodes in the cluster to sleep so as to extend the network lifetime and reduce energy consumption of the entire cluster while keeping a certain fraction of the sensors energy-balanced?

In order to balance the energy consumption of a large fraction of the sensor nodes in a cluster, we need to manipulate the sleeping probability of each sensor node according to its distance from the cluster head. However, unlike the DS scheme where the only criterion was to choose the sleeping probabilities to reduce overall energy consumption, the goal here is to ensure the average energy consumption of a large number of the nodes is the same. Assuming that the nodes start with approximately the same initial energy, this will ensure that these energy-balanced nodes run out of energy at approximately the same time, thereby extending network lifetime while maintaining adequate sensing coverage. To accomplish this goal, we propose and analyze the Balancedenergy Scheduling (BS) scheme, which is also a distancebased scheme, in this paper. The benefits of the BS scheme will be shown numerically in Section V.

\section{RELATED WORK}

There has been some published work related to the cluster formation and cluster head selection problem [3], [7]. In our work, we study the sleeping node selection problem by assuming that one of these clustering techniques is in use and the clusters and cluster heads are already in place.

Several schemes have been proposed in the literature to determine which nodes should be allowed to sleep. In [4], network nodes are allowed to go to sleep according to routing information and information from the application layer. This paper proposed the Basic Energy Conserving Algorithm (BECA) and the Adaptive Fidelity Energy-Conserving Algorithm (AFECA). In the BECA scheme, nodes switch among sleeping, idling, and active states to save energy. A node alternates between the sleep state and the idling state if no data traffic is present. An idling node goes into the active state when it receives traffic from its application layer or from its neighbors. The AFECA scheme was designed to work with an on-demand routing protocol. In the AFECA scheme, the intervals between consecutive times that a sleeping node wakes up and listens to the channel are a multiple of the route discovery interval, at the end of which Route REQuest (RREQ) packets are transmitted.

Span was proposed in [8] to maximize the amount of time network nodes spend in the sleep state while maintaining the same traffic latency and network capacity. In Span, a few nodes are selected as Coordinators, which do not sleep. All other nodes go into the sleep state according to a sleep/wake cycle specified by the Coordinators. Only the Coordinators participate in packet routing. Since significant energy is consumed by these Coordinators, Span includes a procedure to rotate the Coordinator role among the nodes in the network. Significant energy saving was reported with the help of Span.

In [9], a node-scheduling scheme was proposed to reduce the overall system energy consumption by turning off some redundant nodes in sensor networks. The coverage-based offduty eligibility rule and the backoff-based node-scheduling scheme guarantee that the original sensing coverage area is maintained even after nodes are turned off. According to these rules, sensor nodes can turn themselves off when they notice that their neighbors can cover all of their sensing coverage area. In order to avoid neighboring nodes turning off simultaneously, a back-off based approach was designed.

In the S-MAC scheme [10], energy consumption is reduced by allowing randomly-selected idle sensors to go into the sleep mode. The traffic intended for these sleeping nodes is temporarily stored at the neighboring active nodes. The sleeping sensors wake up periodically to retrieve the stored packets from their neighboring nodes.

In the Energy Dependent Participation (EDP) scheme [11], ad hoc network nodes decide whether to participate in ad hoc routing based on their residual energy. When the residual energy is high, a network node participates in routing with higher probability. This probability is lower when the residual energy is low. A balanced energy consumption is achieved and the extension of network lifetime was reported in the paper.

Some of the schemes discussed above, e.g., [7] and [8], require some knowledge of the entire network before a sensor node can decide to go to sleep. Other schemes such as [4], [9], and [11] make decisions according to a specific system metric such as routing fidelity, sensing coverage, or residual energy. Schemes in [4] and [11] are not suitable for cluster-based sensor networks in which the goal is to improve energy saving while maintaining the same sensing coverage. Other proposed methods, such as those described in [12], [13], and [14], were not designed for cluster-based sensor networks, even though they studied coverage and connectivity in the context of extra sensor nodes in sensor networks. The schemes in [10] and [9] did not consider the variable transmission range of sensor nodes. In the following section, we propose a sleep scheduling scheme that exploits the variable transmission range of sensor nodes to save energy while maintaining the same sensing coverage in cluster-based sensor networks.

In [15], the time and energy costs of both computation and communication activities were considered in the task allocation problems for wireless networked embedded systems with homogeneous elements. In order to extend the network lifetime, the authors' goal is to balance the energy dissipation of the elements during each period of the application with respect to the remaining energy of elements. An optimal solution and a heuristic approach were proposed in the paper. Unlike in [15], we use a probabilistic approach to balance the energy consumption of the sensor nodes while maintaining the 
sensing coverage of the cluster.

\section{The SleEp Scheduling Schemes}

In our study, the following assumptions are made about the sensor network:

- A sufficient number of sensor nodes are deployed over a sensing field such that some sensor nodes can go into the sleeping mode without degrading the sensing coverage of the network.

- Static circular cluster associations are assumed in the sensor network. Each sensor node belongs to the same cluster throughout its lifetime. ${ }^{2}$

- Each sensor can use variable transmission power (assumed to be a continuous variable here) according to its distance from its cluster head [16]. Consequently, it can use the minimal transmission power that is necessary for communication with its cluster head. The cluster head, however, uses the maximum transmission power, with a range of $R$, to communicate with all the sensor nodes. ${ }^{3}$

- The distance between each sensor node and the cluster head is known to these two nodes. The distance can be estimated, e.g., by measuring the strength of signals received from the cluster head. It is not necessary for a node to know other sensors' distances to the cluster head.

- Nodes are randomly distributed as a two-dimensional Poisson point process with density $\rho$. Therefore, the probability of finding $n$ nodes in a region of area $A$ is equal to $(\rho A)^{n} \cdot e^{-\rho A} / n$ !. Furthermore, these $n$ nodes are uniformly distributed in the area.

- $\lambda$ is the average packet transmission rate per second of each sensor node sending data to the cluster head during its non-sleep period, which includes all data transmission periods and idle periods. ${ }^{4}$

We further assume that the energy saving of each sleeping node per second is the expected energy consumption if the node were awake, including the required energy to transmit sensing results to the cluster head and the energy consumed when the node is idle. That is, the average energy consumption per second of the active nodes is

$$
E_{\text {active }}(x)=\lambda \cdot k_{1} \cdot\left[\max \left(x_{\min }, x\right)\right]^{\gamma}+k_{2},
$$

where $k_{1}$ is the constant corresponding to energy consumption due to transmission of each packet, $k_{2}$ is the idle/receive energy consumption per second, $x_{\min }$ is the minimum transmission range corresponding to the minimum allowable transmission energy [17], and $\gamma \geq 2$ is the path loss exponent. The max function indicates that, even if the distance between

\footnotetext{
${ }^{2}$ The cluster head might be rotated among nodes in a small region near the center of the cluster, so that the distance between each sensor node and the cluster head stays approximately the same.

${ }^{3}$ Although a multi-hop cluster structure is possible, it will significantly increase the intra-cluster communication overhead and management task for the cluster. A discussion of the advantages and disadvantages of such a multihop approach is out of the scope of this work.

${ }^{4}$ The sleeping nodes do not generate any traffic to send to the cluster head. However, we stress that the neighborhoods of the sleeping nodes are covered by other active neighboring sensors [6].
}

a sensor node and the cluster head is smaller than $x_{\text {min }}$, the sensor needs to spend the energy that corresponds to $x_{\min }$ for its transmission. We further assume that the initial energies of all nodes are the same.

\section{A. The RS and the DS Schemes}

In order to save energy and extend the network lifetime as long as possible, some extra sensors may be put into the sleep state, in which these sensor nodes consume much less energy. It is, however, non-trivial to select a fraction of these nodes to sleep, as the selection of different sensors may affect the performance of the entire cluster. More specifically, the total energy consumption and sensing coverage may be affected depending on which sensors are active and which are asleep. In [6], we studied the Sleep Scheduling problem, as described in Section I. We generalized and proposed two sleep scheduling schemes, termed the Randomized Scheduling (RS) scheme and the Distance-based Scheduling (DS) scheme. A brief introduction of these two schemes is provided below. Detailed discussions on the energy saving and sensing coverage of these two schemes may be found in [6].

In the RS scheme, the sleeping sensor nodes are selected randomly from among the nodes in the cluster. Assuming the average fraction of sensors allowed to sleep is $\beta_{s}<1$, each sensor node goes into the sleep state with probability $p=\beta_{s}$.

In the DS scheme, however, the probability that a node goes into the sleep state, $p$, is related to the distance between the sensor and its cluster head, $x$. A sensor node that is farther away from the cluster head will be put into the sleep state with higher probability. Energy can be saved by allowing nodes that are far from the cluster head to sleep compared with allowing nodes closer to the cluster head to sleep. The sleeping probability of a sensor node in the DS scheme is (when $\beta_{s}<\frac{2}{3}$ )

$$
p(x)=\frac{3 R \beta_{s}}{4} \cdot \frac{2 x}{R^{2}}=\frac{3 \beta_{s} x}{2 R} \quad 0 \leq x \leq R .
$$

\section{B. Coefficient of Variation of Energy Consumption}

Intuitively, when the sensor nodes consume approximately the same amount of energy per second, they run out of energy at about the same time and there will not be any holes in the cluster due to dead sensors during network lifetime. In this subsection, we analyze the coefficient of variation of sensor nodes' energy consumption when the RS or the DS scheme is employed. We present the studies on their network lifetime in Section V-C.

When the RS scheme is employed, each node goes to sleep in each cycle ${ }^{5}$ with probability $p=\beta_{s}$. Therefore, the expected energy consumption per second of a sensor node that is a distance $x$ from the cluster head is:

$$
E_{R S}(x)=\left(1-\beta_{s}\right) E_{\text {active }}(x) \quad 0 \leq x \leq R .
$$

\footnotetext{
${ }^{5}$ The exact length of a cycle is left for system implementation. However, we want to point out that a small cycle duration increases the overhead of a sleeping scheme. On the other hand, a large cycle duration may reduce the impact of a sleeping scheme.
} 
The expected energy consumption per second per sensor node can be calculated as:

$$
\begin{aligned}
& E_{R S} \\
= & \int_{0}^{R}\left(1-\beta_{s}\right) E_{\text {active }}(x) \cdot f(x) d x \\
= & \frac{1-\beta_{s}}{R^{2}}\left\{\frac{\lambda k_{1} \gamma}{\gamma+2}\left(x_{\text {min }}\right)^{\gamma+2}+\frac{2 \lambda k_{1}}{\gamma+2} R^{\gamma+2}+k_{2} R^{2}\right\}
\end{aligned}
$$

where $f(x)=\frac{2 x}{R^{2}}, 0 \leq x \leq R$, is the Probability Density Function (PDF) of the distance, $x$, between a sensor and the cluster head, based on the assumption that the sensor nodes are distributed uniformly in the circular cluster region.

The variance of the energy consumption of the sensor nodes is $\sigma_{R S}^{2}$ :

$$
\begin{aligned}
& \sigma_{R S}^{2}=\int_{0}^{R} f(x)\left[E_{R S}(x)-E_{R S}\right]^{2} d x \\
= & \left(1-\beta_{s}\right)^{2}\left\{\frac{\left(x_{\text {min }}\right)^{2}}{R^{2}} \cdot\left[\lambda k_{1}\left(x_{\text {min }}\right)^{\gamma}+k_{2}\right]^{2}\right. \\
+ & \frac{2}{R^{2}} \cdot\left\{\frac{\left(\lambda k_{1}\right)^{2}}{2 \gamma+2}\left[R^{2 \gamma+2}-\left(x_{\text {min }}\right)^{2 \gamma+2}\right]\right. \\
+ & \left.\frac{2 \lambda k_{1} k_{2}}{\gamma+2}\left[R^{\gamma+2}-\left(x_{\text {min }}\right)^{\gamma+2}\right]+\frac{\left(k_{2}\right)^{2}}{2}\left[R^{2}-\left(x_{\text {min }}\right)^{2}\right]\right\} \\
- & \left.\frac{1}{R^{4}}\left[\frac{\lambda k_{1} \gamma}{\gamma+2}\left(x_{\text {min }}\right)^{\gamma+2}+\frac{2 \lambda k_{1}}{\gamma+2} R^{\gamma+2}+k_{2} R^{2}\right]^{2}\right\} .
\end{aligned}
$$

The coefficient of variation of energy consumption is then $c v_{R S}=\sqrt{\sigma_{R S}^{2}} / E_{R S}$. Note that $c v_{R S}$ is not related to $\beta_{s}$ since the terms $\left(1-\beta_{s}\right)$ in the numerator and the denominator cancel out.

When the DS scheme is employed, every sensor node goes to sleep based on the probability $p(x)$ as expressed in (2). Similar to (3), the expected energy consumption per second of a sensor node that is a distance $x$ away from the cluster head is:

$$
\begin{aligned}
E_{D S}(x) & =[1-p(x)] E_{\text {active }}(x) \\
& =\left(1-\frac{3 \beta_{s} x}{2 R}\right) \cdot E_{\text {active }}(x),
\end{aligned}
$$

where $0 \leq x \leq R$. The expected value of energy consumption is:

$$
\begin{aligned}
E_{D S}= & \int_{0}^{R}[1-p(x)] E_{\text {active }}(x) \cdot f(x) d x \\
= & \frac{1}{R^{2}}\left[\frac{\lambda k_{1} \gamma}{\gamma+2}\left(x_{\text {min }}\right)^{\gamma+2}+\frac{2 \lambda k_{1}}{\gamma+2} R^{\gamma+2}+k_{2} R^{2}\right] \\
& -\frac{\beta_{s}}{R^{3}}\left[\frac{\lambda k_{1} \gamma}{\gamma+3}\left(x_{\text {min }}\right)^{\gamma+3}+\frac{3 \lambda k_{1}}{\gamma+3} R^{\gamma+3}+k_{2} R^{3}\right](6)
\end{aligned}
$$

Similarly, for the DS scheme, the variance of the sensor nodes' energy consumption, $\sigma_{D S}^{2}$, becomes: ${ }^{6}$

$$
\sigma_{D S}^{2}=\int_{0}^{R} f(x)\left[E_{D S}(x)-E_{D S}\right]^{2} d x
$$

\footnotetext{
${ }^{6}$ Due to page limitations, we omit the closed form of this equation.
}

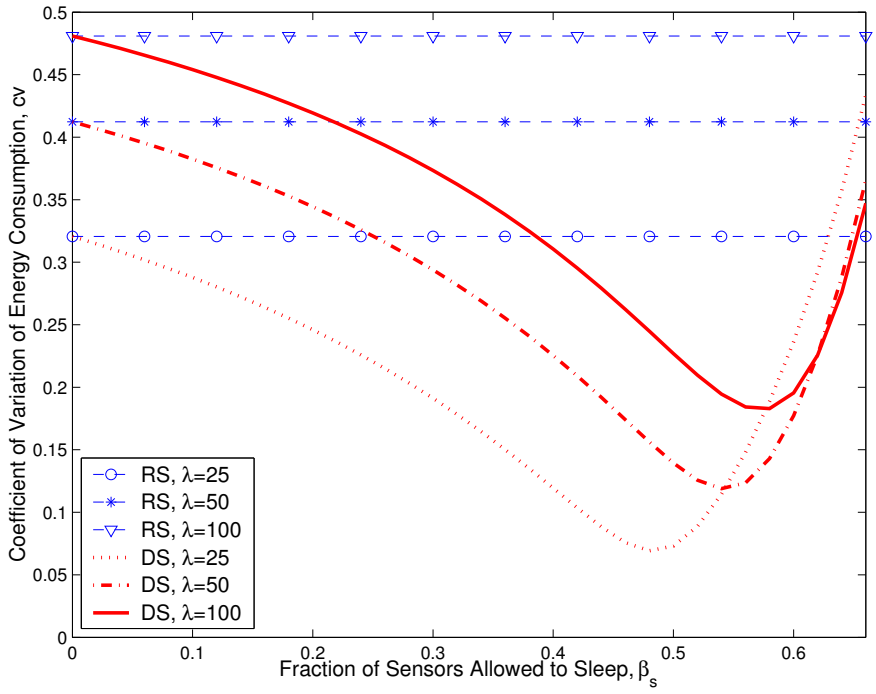

Fig. 1. Coefficient of Variation of the Sensor Nodes' Energy Consumption, $c v$.

The coefficient of variation is $c v_{D S}=\sqrt{\sigma_{D S}^{2}} / E_{D S}$.

In Fig. 1, we draw the coefficient of variation of the sensor nodes' energy consumption for the RS and the DS schemes. In the sensor network that we studied, we assume that there are $N=500$ sensors in each cluster, $k_{1}=10^{-6} \mathrm{~J} /$ (packet . $\left.\mathrm{m}^{2}\right), k_{2}=0.1 \mathrm{~J} / \mathrm{sec}$, and $x_{\min }=10 \mathrm{~m}$. The traffic load on each active sensor node $\lambda$ takes on the values of 25,50 , and $100 \mathrm{packet} / \mathrm{sec}$ to demonstrate different energy consumption requirements. The maximum transmission range of the cluster head is $R=100 \mathrm{~m}$. The path loss exponent is $\gamma=2$.

As mentioned before, $c v_{R S}$ is not related to $\beta_{s}$. However, $c v_{R S}$ increases with an increase in $\lambda$. For example, $c v_{R S}$ is 0.32 when $\lambda$ is 25 packets/sec while $c v_{R S}$ becomes 0.48 when traffic load $\lambda$ increases to 100 packets/sec. This increase could be due to the larger relative energy consumption for nodes on the border of the circular cluster region. Interestingly, $c v_{D S}$ decreases with an increase of the expected sleeping probability, $\beta_{s}$, until $\beta_{s}$ reaches between 0.5 and 0.6 , depending on $\lambda$, and then it increases with $\beta_{s} . c v_{D S}$ is generally lower than the corresponding $c v_{R S}$, as the DS scheme allows the farther-away nodes, which need to spend more energy to transmit to the cluster head, to sleep with higher probability. This can be explained in the following intuitive way: the RS scheme selects sensor nodes to sleep randomly. However, the sensor nodes that are farther away from the cluster head consume much higher energy than those that are closer to the center of the cluster. Therefore, the energy consumptions of nodes from different regions vary significantly. In the DS scheme, the farther-away nodes are selected to sleep with higher probability, leading to more balanced energy consumption among all sensor nodes. In the following section, we propose a scheme to further lower the coefficient of variation of the energy consumption of sensor nodes. 


\section{BALANCED-Energy Scheduling (BS) Scheme}

In the Balanced-energy Scheduling (BS) scheme, a sleeping probability $p(x)$ is chosen in such a way that as many sensor nodes as possible consume the same amount of energy, on average. Therefore, the BS scheme is actually a special case of the DS scheme. Let $E_{B S}(x)$ be the expected energy consumption of a node at a distance $x$ from the cluster head. Our goal is to find a $p(x)$ such that $E_{B S}(x)$ does not depend on the value of $x$ :

$E_{B S}(x)=[1-p(x)] E_{\text {active }}(x)=E_{B S}^{(b)} \quad$ for all $x_{b} \leq x \leq R$

where the use of $x_{b}$ guarantees that $p(x) \geq 0$, as $E_{a c t i v e}(x)$ is a non-decreasing function of $x$. Note that the nodes close to the cluster head might not be energy-balanced with other nodes, as their energy consumption per transmission is much smaller than others based on (1). However, we should minimize $E_{B S}^{(b)}$ when a feasible $x_{b}$ is given. Since another important goal of the sleep scheduling scheme is to save as much energy as possible, we should let those sensor nodes that are closer than $x_{b}$ to the cluster head remain awake all the time (for a fixed $\beta_{s}$ ). Therefore, we have

$$
p(x)=\left\{\begin{array}{ll}
1-\frac{E_{B S}^{(b)}}{E_{\text {active }}(x)} \geq 0 & \text { for all } x_{b} \leq x \leq R \\
0 & \text { otherwise }
\end{array} .\right.
$$

The feasible range of $x_{b}$ will be determined later. It can be proven that $E_{B S}^{(b)}$ is a non-increasing function of $x_{b}$ for a fixed $\beta_{b}$.

In (8), the value of $E_{B S}^{(b)}$ is related to the fraction, $\beta_{s}$, of sensor nodes that are allowed to sleep:

$\int_{0}^{R} p(x) \cdot f(x) d x=\int_{x_{b}}^{R}\left(1-\frac{E_{B S}^{(b)}}{E_{\text {active }}(x)}\right) \frac{2 x}{R^{2}} d x=\beta_{s}$.

The above equation allows us to determine the relation between $E_{B S}^{(b)}$ and $\beta_{s}$ :

$$
\begin{aligned}
E_{B S}^{(b)} & =\frac{R^{2}\left(1-\beta_{s}\right)-x_{b}^{2}}{2 \int_{x_{b}}^{R} \frac{x}{E_{a c t i v e}(x)} d x} \\
& =\frac{R^{2}\left(1-\beta_{s}\right)-x_{b}^{2}}{2 \int_{x_{b}}^{R} \frac{x}{\lambda k_{1}\left[\max \left(x_{\min }, x\right)\right]^{\gamma}+k_{2}} d x} .
\end{aligned}
$$

Since $E_{B S}^{(b)}$ should not be less than 0 , we can derive the upper bound on $x_{b}$ as

$$
x_{b} \leq R \sqrt{1-\beta_{s}} .
$$

Also, since $x_{b}$ should guarantee that $p(x) \geq 0$ and notice from (8) that $p(x)$ increases with $x_{b}$, a lower bound of $x_{b}$ should satisfy

$$
p\left(x=x_{b}\right)=1-\frac{E_{B S}^{(b)}}{E_{\text {active }}\left(x_{b}\right)} \geq 0,
$$

which means $x_{b}$ and $E_{B S}^{(b)}$ should satisfy

$$
E_{B S}^{(b)} \leq \lambda k_{1}\left[\max \left(x_{\min }, x_{b}\right)\right]^{\gamma}+k_{2} .
$$

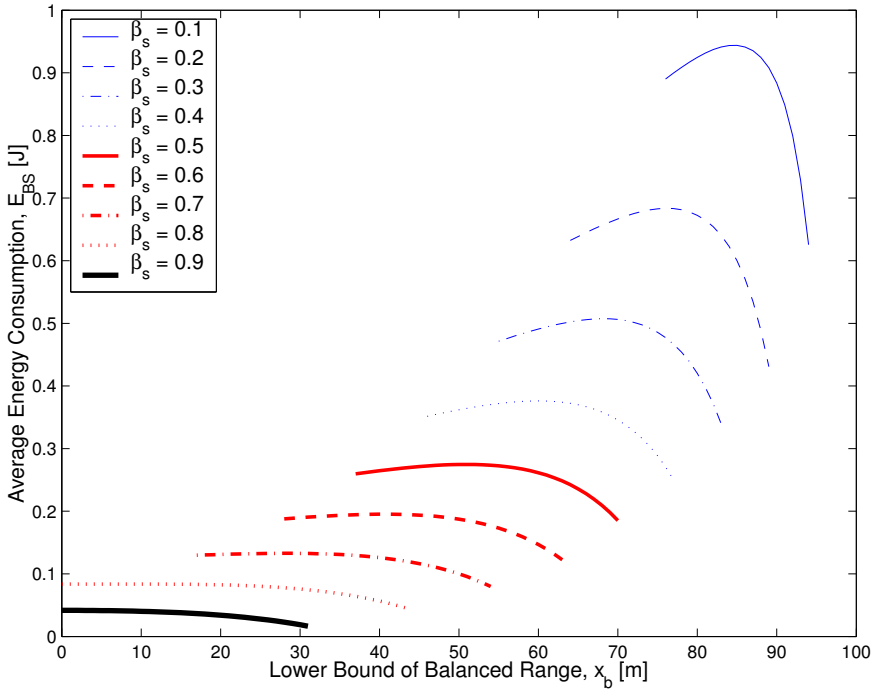

Fig. 2. Energy Consumption of the BS Scheme for Different $\beta_{s}(\gamma=2)$.

It can be proven that if $x_{b}=x_{\min }$ satisfies the above inequality, then $x_{b}$ can be set to 0 .

When a BS scheme is employed as given by (8), the fraction of sensors that are energy-balanced, $\beta_{b}$, can be calculated as:

$$
\beta_{b}=\frac{n \cdot\left[1-\int_{0}^{x_{b}} f(x) d x\right]}{n}=1-\frac{x_{b}^{2}}{R^{2}} .
$$

Thus, the value of $\beta_{b}$ increases as $x_{b}$ decreases. In order to increase the fraction of sensors that are energy balanced, we should decrease $x_{b}$. Unfortunately, the decrease of $x_{b}$ in its allowable range leads to an increase of the expected energy consumption of a sensor node, as shown in (9).

Based on $f(x)$, the expected energy consumption of a sensor node can be calculated as the average over the entire cluster:

$$
E_{B S}=\int_{0}^{x_{b}} E_{\text {active }}(x) \frac{2 x}{R^{2}} d x+E_{B S}^{(b)} \frac{R^{2}-x_{b}^{2}}{R^{2}} .
$$

Figure 2 presents the average energy consumption of the BS scheme for different average fraction of nodes that are allowed to sleep, $\beta_{s}$. In this figure, we draw the expected energy consumption of a sensor node, $E_{B S}$ in (13), for the range of allowable $x_{b}$, which satisfies (10) and (11). As shown in the figure, the allowable range of $x_{b}$ is relatively small given a fixed $\beta_{s}$. We can also observe that, when $\beta_{s}$ is small, the upper bound of the feasible ranges of $x_{b}$ should be selected, which minimizes the average energy consumption. However, by noticing that $\beta_{b}=1-\frac{x_{b}^{2}}{R^{2}}$, when $\beta_{s}$ becomes larger, e.g., 0.45 to 0.9 , it might be more appropriate to select the lower bound of the $x_{b}$ values. Even though this selection may lead to slightly higher energy consumption, it results in a much larger fraction of sensor nodes that are energy-balanced.

\section{Performance Evaluation}

In this section, we study the performance of the BS scheme, including its average energy consumption, coefficient of variation of energy consumption, sensing coverage, and network lifetime. 


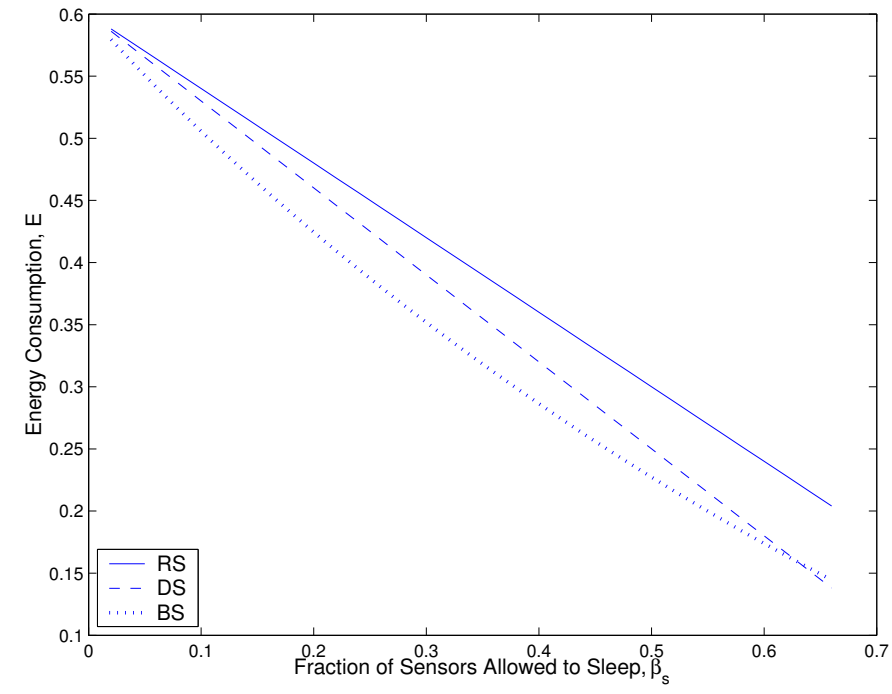

Fig. 3. Energy Consumption Comparison of the RS, DS, and BS schemes $(\gamma=2)$.

\section{A. Average Energy Consumption}

The average energy consumption of the BS scheme can be calculated by (13):

$$
\begin{aligned}
E_{B S} & =\left[\lambda k_{1}\left(x_{\text {min }}\right)^{\gamma}+k_{2}\right] \cdot \frac{\left(x_{1}\right)^{2}}{R^{2}} \\
& +\frac{2 \lambda k_{1}\left[\left(x_{2}\right)^{\gamma+2}-\left(x_{\min }\right)^{\gamma+2}\right]}{(\gamma+2) R^{2}} \\
& +\frac{k_{2}\left[\left(x_{2}\right)^{2}-\left(x_{\min }\right)^{2}\right]}{R^{2}}+E_{B S}^{(b)} \frac{R^{2}-x_{b}^{2}}{R^{2}},
\end{aligned}
$$

where $x_{1}$ and $x_{2}$ are

$$
x_{1}=\min \left(x_{b}, x_{\text {min }}\right) \text { and } x_{2}=\max \left(x_{b}, x_{\text {min }}\right),
$$

and $E_{B S}^{(b)}$ is given by (9):

$$
E_{B S}^{(b)}=\frac{R^{2}\left(1-\beta_{s}\right)-x_{b}^{2}}{\frac{\left(x_{\min }\right)^{2}-\left(x_{1}\right)^{2}}{\lambda k_{1}\left(x_{m i n}\right)^{\gamma}+k_{2}}+2 \int_{x_{2}}^{R} \frac{x}{\lambda k_{1} x^{\gamma}+k_{2}} d x} .
$$

A closed form is available for the integral in (16) when $\gamma=2,3$, and 4 . Due to page limitations, we only present the closed form when $\gamma=2$ :

$$
2 \int_{x_{2}}^{R} \frac{x}{\lambda k_{1} x^{\gamma}+k_{2}} d x=\frac{1}{\lambda k_{1}} \ln \left[\frac{\lambda k_{1} R^{2}+k_{2}}{\lambda k_{1}\left(x_{2}\right)^{2}+k_{2}}\right] .
$$

Combining (17) with (16) and substituting in (14), we have a closed form solution for the average energy consumption for the BS scheme when $\gamma=2$.

In Fig. 3, we show the average energy consumption of the $\mathrm{RS}$, the DS, and the BS schemes. The traffic load $\gamma$ is fixed at $100 \mathrm{packet} / \mathrm{sec}$ in this figure. We select $x_{b}$ as the lower bound in (11) in order to maximize the fraction of sensor nodes that are energy-balanced. As expected, the average energy consumption of all three schemes decreases with an increase of $\beta_{s}$. This figure shows that the average energy consumption of the DS and the BS schemes is always lower than that of

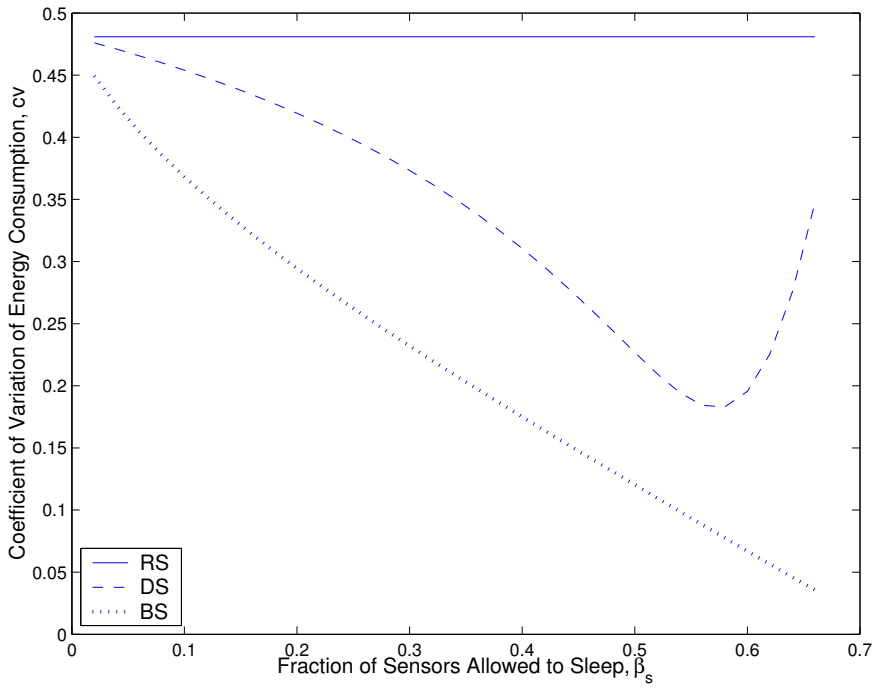

Fig. 4. Coefficient of Variation Comparison of the RS, DS, and BS schemes $(\gamma=2)$

the RS scheme. The BS scheme out-performs the DS scheme in average energy consumption for most of the values of $\beta_{s}$ we show.

\section{B. Coefficient of Variation of Energy Consumption}

When the BS scheme is employed, the variance of the sensor nodes' energy consumption becomes

$$
\begin{aligned}
& \sigma_{B S}^{2} \\
= & \int_{0}^{R} f(x)\left[E_{B S}(x)-E_{B S}\right]^{2} d x \\
= & {\left[\lambda k_{1}\left(x_{m i n}\right)^{\gamma}+k_{2}\right]^{2} \frac{\left(x_{1}\right)^{2}}{R^{2}} } \\
+ & 2\left(\lambda k_{1}\right)^{2} \frac{\left(x_{2}\right)^{2 \gamma+2}-\left(x_{m i n}\right)^{2 \gamma+2}}{(2 \gamma+2) R^{2}} \\
+ & 4 \lambda k_{1} k_{2} \frac{\left(x_{2}\right)^{\gamma+2}-\left(x_{m i n}\right)^{\gamma+2}}{(\gamma+2) R^{2}} \\
+ & \left(k_{2}\right)^{2} \frac{\left(x_{2}\right)^{2}-\left(x_{m i n}\right)^{2}}{R^{2}}+\left(E_{B S}^{(b)}\right)^{2} \frac{R^{2}-x_{b}^{2}}{R^{2}}-\left(E_{B S}\right)^{2},
\end{aligned}
$$

where $x_{1}$ and $x_{2}$ are given by (15), $E_{B S}^{(b)}$ is given by (16), $E_{B S}(x)$ is the energy consumption of a sensor node that is $x$ away from the cluster head (e.g., $E_{B S}(x)=E_{B S}^{(b)}$ for $x>x_{b}$ ), and $E_{B S}$ is given by (14). Coefficient of variation is then $c v_{B S}=\sqrt{\sigma_{B S}^{2}} / E_{B S}$.

In Fig. 4, we show the coefficient of variation of the energy consumption of sensor nodes when the DS, the RS, and the BS schemes are employed, respectively. Again, $x_{b}$ is selected as shown in (11), and $\lambda=100$ packets/sec. $c v_{B S}$ is lower than $c v_{R S}$ and $c v_{D S}$, as shown in the figure. Therefore, the energy consumption of the BS scheme is more balanced. The values of $c v_{B S}$ decrease with an increase of $\beta_{s}$ because the lower bound of $x_{b}$ ranges is smaller for larger $\beta_{s}$, such that more nodes are energy-balanced (i.e., larger $\beta_{b}$ ). 


\section{Network Lifetime}

We define the network lifetime $T\left(\beta_{d}\right)$ as the time when a fraction of sensors, $\beta_{d}$, run out of energy. Let $\Psi$ be the total battery energy each sensor node carries when the sensor network is initialized. Since the cluster coverage drops below $90 \%$ when $\beta_{s}>0.4$ for the parameters used in our scenario (see section V-D), we compare the lifetime of the three sleep scheduling schemes for $\beta_{s}<0.4$.

In the BS scheme, all nodes with distance $x \geq x_{b}$ from the cluster head run out of energy at the same time, as they consume the same energy on average. In order to simplify the discussion, we only consider the case when $x_{b}$ is chosen to be the smallest value of its allowable range. Consequently, all sensor nodes that are closer than $x_{b}$ to the cluster head consume less energy than $E_{B S}^{(b)}$. Furthermore, $x_{b}$ satisfies either $x_{b}>x_{\min }$ or $x_{b}=0$.

Since a fraction of $\beta_{b}$ sensor nodes consume the same energy on the average, when $\beta_{d} \leq \beta_{b}$,

$$
T_{B S}\left(\beta_{d}\right)=\frac{\Psi}{E_{B S}^{(b)}},
$$

where $E_{B S}^{(b)}$ is given by (16).

When $\beta_{d}>\beta_{b}$, we should consider the time when a fraction of $\beta_{d}-\beta_{b}$ sensors located at distance $x, x_{\min }<x<x_{b}$, from the cluster head run out of energy. Since all sensor nodes at distance less than $x_{\min }$ from the cluster head will consume the same energy, when

$$
\beta_{d}>\beta_{b}+\int_{x_{\min }}^{x_{b}} f(x) d x=\beta_{b}+\frac{x_{b}^{2}-x_{\min }^{2}}{R^{2}},
$$

the network lifetime is

$$
T_{B S}\left(\beta_{d}\right)=\frac{\Psi}{E_{\text {active }}\left(x_{\min }\right)}=\frac{\Psi}{\lambda k_{1}\left(x_{\min }\right)^{\gamma}+k 2} .
$$

When $\beta_{b}<\beta_{d} \leq \beta_{b}+\frac{x_{b}^{2}-x_{\min }^{2}}{R^{2}}$, all the energy-balanced sensor nodes and another $\beta_{d}-\beta_{b}$ portion of sensor nodes run out of energy in $T_{B S}\left(\beta_{d}\right)$. We have

$$
T_{B S}\left(\beta_{d}\right)=\frac{\Psi}{E_{\text {active }}\left(x_{d}^{(B S)}\right)}=\frac{\Psi}{\lambda k_{1}\left[x_{d}^{(B S)}\right]^{\gamma}+k 2},
$$

where $x_{d}^{(B S)}=\sqrt{x_{b}^{2}-\left(\beta_{d}-\beta_{b}\right) R^{2}}$.

In the RS scheme, however, the sensor nodes farther away from the cluster head consume much more energy than the sensor nodes that are closer to the cluster head due to (1). Therefore, the outer sensor nodes will run out of energy much faster than the inner sensor nodes. The time when $\beta_{d}$ fraction of nodes run out of energy is the time when sensor nodes with $x \geq x_{d}^{(R S)}$ all run out of energy, where $x_{d}^{(R S)}$ satisfies:

$$
\beta_{d}=\int_{x_{d}^{(R S)}}^{R} f(x) d x=\frac{R^{2}-\left[x_{d}^{(R S)}\right]^{2}}{R^{2}},
$$

leading to $x_{d}^{(R S)}=R \cdot \sqrt{1-\beta_{d}}$.

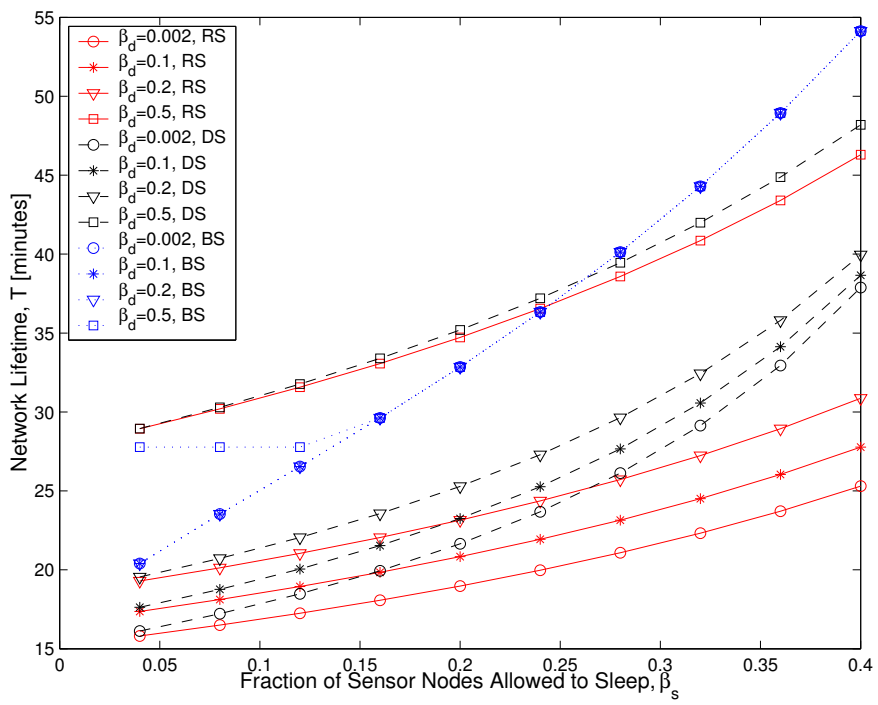

Fig. 5. Comparison of network lifetime RS, DS, and BS schemes $(\gamma=2)$.

The network lifetime of the RS scheme is then

$$
\begin{aligned}
& T_{R S}\left(\beta_{d}\right) \\
= & \frac{\Psi}{E_{R S}\left(x_{d}^{(R S)}\right)} \\
= & \frac{\Psi}{\left(1-\beta_{s}\right)\left\{\lambda k_{1}\left[\max \left(R \cdot \sqrt{1-\beta_{d}}, x_{m i n}\right)\right]^{2}+k_{2}\right\}} .
\end{aligned}
$$

The network lifetime of the DS scheme can be calculated numerically in the following way: from (5), the energy consumption of all sensor nodes can be calculated based on their distance from the cluster head. We then find a $\beta_{d}$ fraction of sensor nodes that run out of energy sooner than the rest of $1-\beta_{d}$ fraction of sensor nodes. The time when the last of these $\beta_{d}$ fraction of sensor nodes runs out of energy represents the network lifetime, $T_{D S}\left(\beta_{d}\right)$.

We show the network lifetime of the RS, the DS, and the BS schemes in Fig. 5. In the calculations, we assume $\Psi=10^{3} \mathrm{J.}^{7}$ The network lifetimes of all three schemes improve as $\beta_{s}$ increases, due to increasing energy saving in the sensor network. The network lifetime of the BS scheme is the same for smaller $\beta_{d}$ because more than $\beta_{d}$ fraction of the sensor nodes are energy-balanced. These nodes run out of energy at approximately the same time. The network lifetime of the RS scheme is shorter than that of the DS scheme. The best network lifetime of the three schemes is that of the BS scheme, except when $\beta_{d}=0.5$ and $\beta_{s}<0.27$. As shown in Fig. 2, when $\beta_{s}$ is smaller, the fraction of sensor nodes that are energy-balanced is smaller in the BS scheme. Therefore, the time that $50 \%$ of the sensor nodes run out of energy is shorter in the BS scheme, resulting in shorter lifetime than the RS and DS schemes when $\beta_{s}<0.27$ and $\beta_{d}=0.5$. As Fig. 5 shows, the $\beta_{d}=0.1$ network lifetime (defined as the

\footnotetext{
${ }^{7}$ These results only have relative significance, as network lifetime depends largely on $\Psi, k_{1}, k_{2}, \gamma$, and other system parameters.
} 


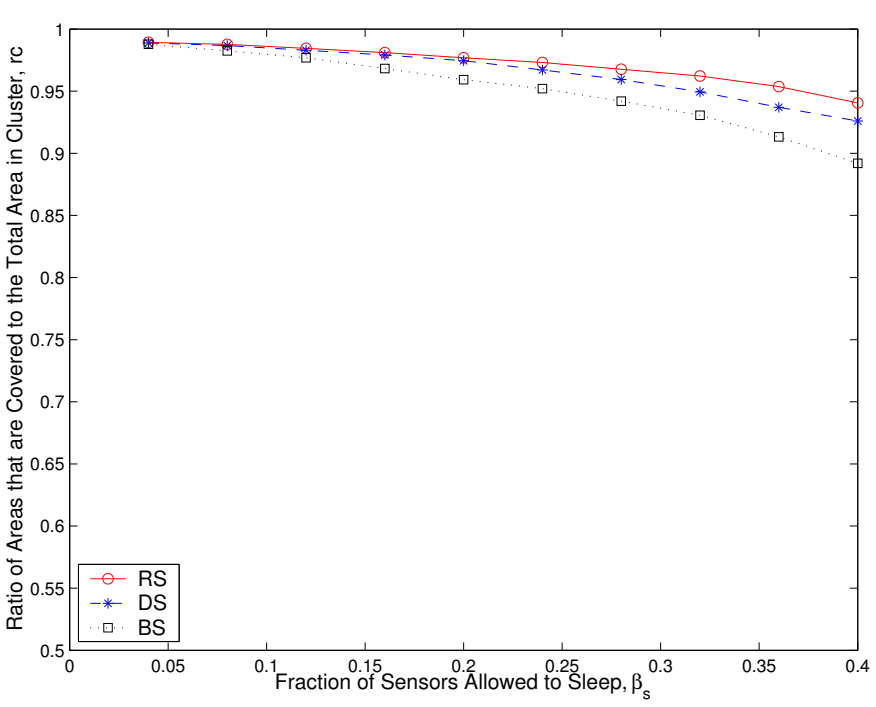

Fig. 6. Comparison of sensing coverage of RS, DS, and BS schemes $(\gamma=2)$.

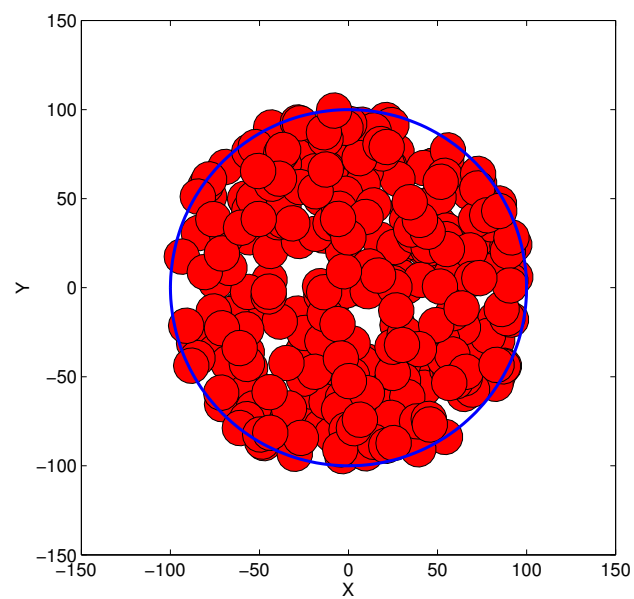

Fig. 7. Areas covered by active nodes in the RS scheme.

time when 50 nodes die, as $N=500$ ), of the BS scheme out-performs the DS and the RS schemes by $70 \%$ and $150 \%$, respectively, when $\beta_{s}$ is close to 0.4 .

\section{Sensing Coverage}

We study the sensing coverage of the BS scheme by means of simulation. Figure 6 compares the sensing coverage performance of the RS, the DS, and the BS schemes. In this figure, we show the ratio of areas in the cluster that are covered by at least one active sensor. The sensing range of each sensor is fixed at $10 \mathrm{~m}$, compared with the $100 \mathrm{~m}$ cluster range, $R$. There are 500 sensors in the cluster. It can be seen that the sensing coverage of the RS scheme is slightly better than that of the DS scheme, which, in turn, out-performs the BS scheme. This is due to the way the sensors are selected to sleep in the DS and the BS schemes. Overall, the sensing coverage of the three schemes are very similar, providing at least $90 \%$ sensing coverage to the cluster when $\beta_{s}<0.4$.

In Figs. 7, 8, and 9, we show snapshots of the cluster

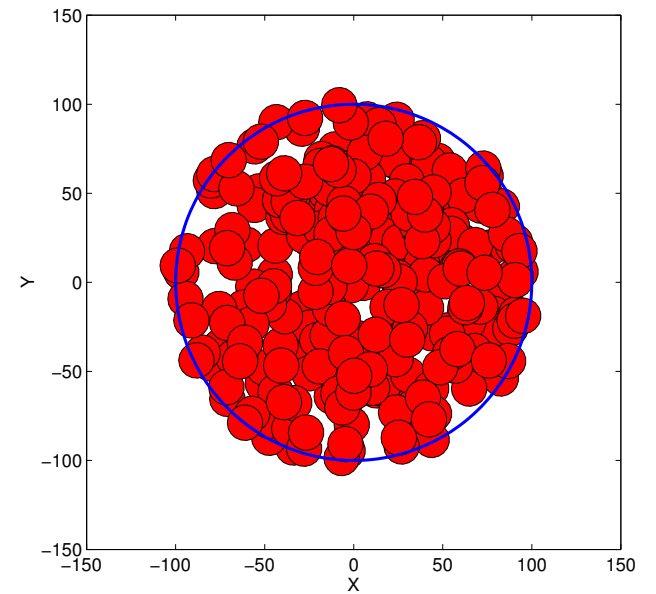

Fig. 8. Areas covered by active nodes in the DS scheme.

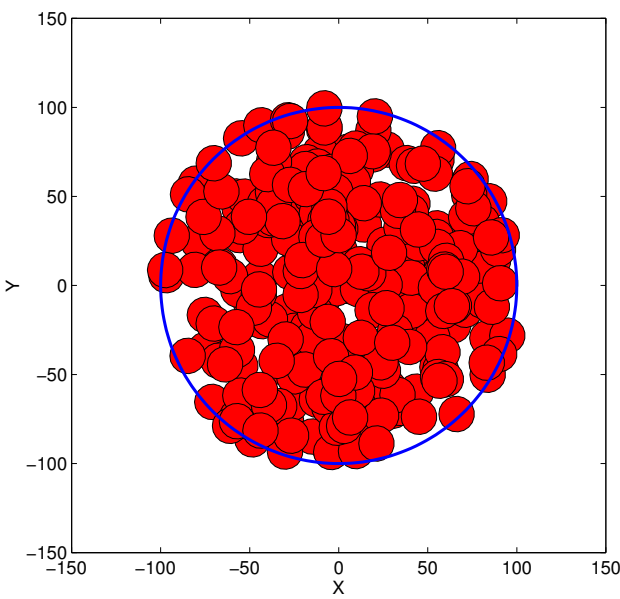

Fig. 9. Areas covered by active nodes in the BS scheme.

coverage when the RS, the DS, or the BS scheme is used. The total number of sensors is 500 and $\beta_{s}$ is 0.4 . The shaded areas represent the areas that are covered by active sensor nodes when different schemes are used to select $\beta_{s}$ portion of sensor nodes to sleep. Note that the total area not covered by any active sensors in all three schemes is about $10 \%$ of the entire circular cluster region, as indicated in Fig. 6. From these three figures, we can see that the regions left uncovered in the cluster with the RS, the DS, and the BS schemes do not differ significantly.

In order to evaluate the uniform-ness of the sensing coverage of the sleep scheduling schemes, we have simulated and recorded the average ratio of coverage in the ring with radius of $r$ from the center of the circular cluster region. We show this ratio of areas being covered in Fig. 10, which represents an average of 20 runs. A perfectly uniform distributed sensing coverage would result in a horizontal line in the figure. However, due to the randomness and the border effect, such a horizontal line cannot be achieved in practice. From Fig. 10, we can see that the RS scheme does provide more uniform sensing coverage except in the border area, while the DS and 


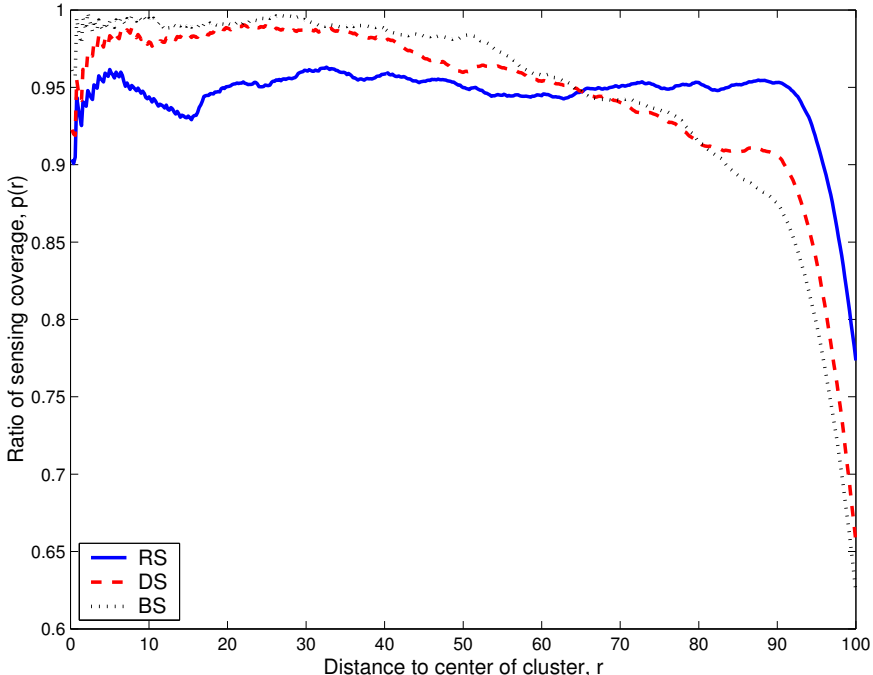

Fig. 10. Sensing coverage distribution for the RS, the DS, and the BS schemes.

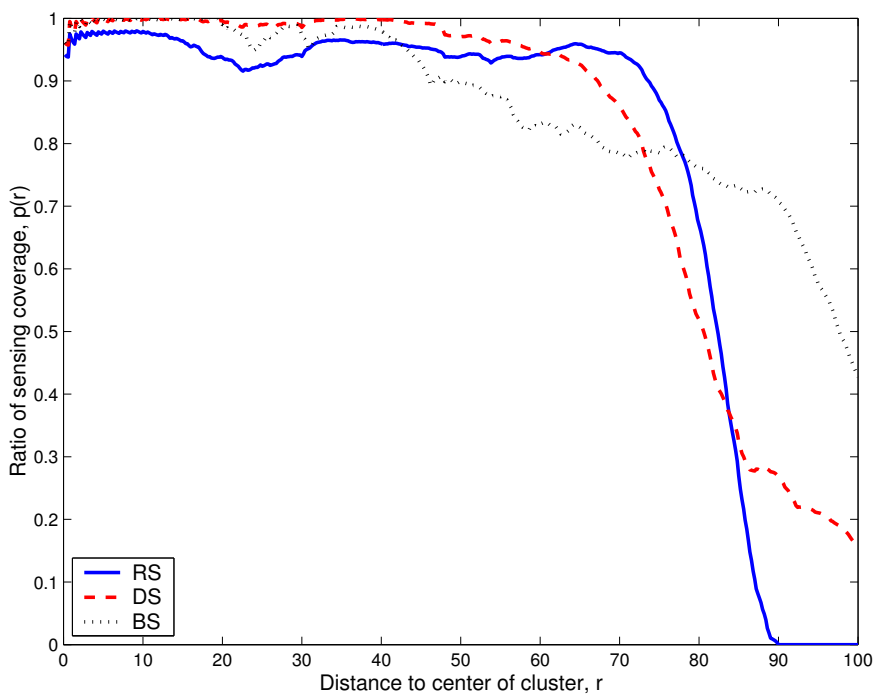

Fig. 11. Sensing coverage distribution for the RS, the DS, and the BS schemes after $40 \%$ of nodes run out of energy.

the BS schemes provide 5-10\% lower sensing coverage in the outer ring of the cluster region. In Fig. 11, we show the ratios after $40 \%$ of sensor nodes run out of energy. While the coverage of the RS scheme is clearly lowered on the border of the region, the BS scheme maintains similar coverage.

Figures 12, 13, and 14 present snapshots of the cluster after $50 \%$ of the sensor nodes run out of energy, when the RS, the DS, and the BS schemes are used, respectively. The small circles represent alive sensor nodes, while the small dots identify the dead sensor nodes. In Fig. 12, the results for the RS scheme, all the dead sensors are in the outside region of the circular cluster region. This is due to the higher energy consumption of these sensor nodes and the pure random selection in the RS scheme. Thus, only the sensors inside a certain radius still have battery energy remaining. Similarly,

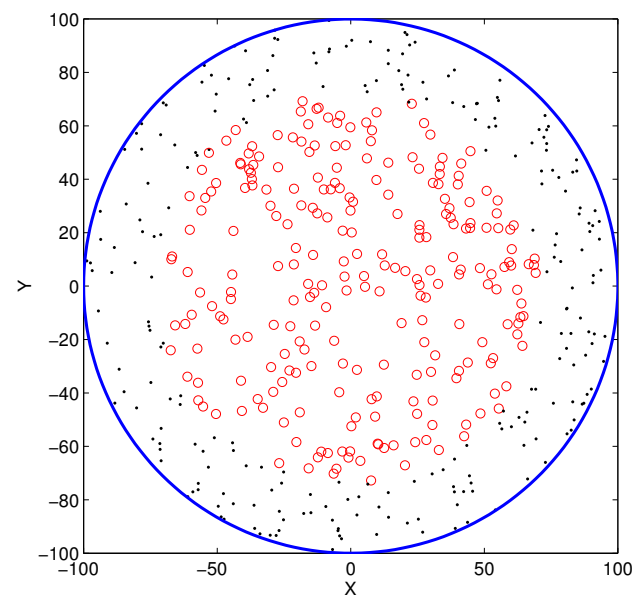

Fig. 12. Sensors that remain alive in the RS scheme after $50 \%$ of the sensor nodes run out of energy. Small circles represent alive sensors nodes, small dots represent dead sensor nodes.

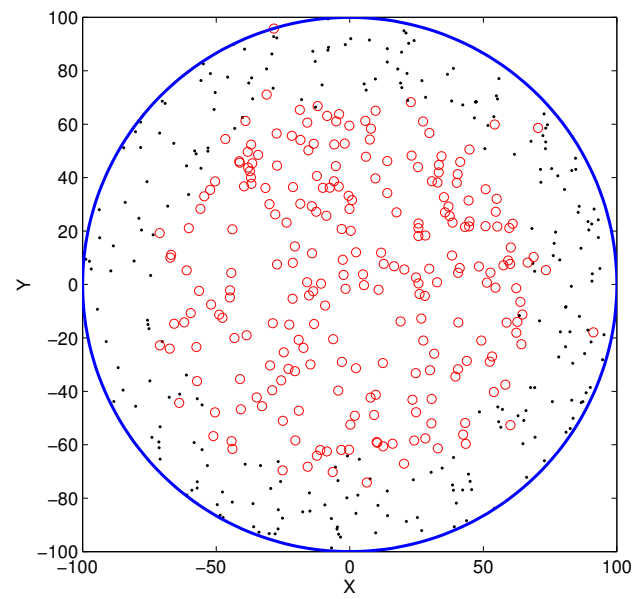

Fig. 13. Sensors that remain alive in the DS scheme after $50 \%$ of the sensor nodes run out of energy. Small circles represent alive sensor nodes, small dots represent dead sensor nodes.

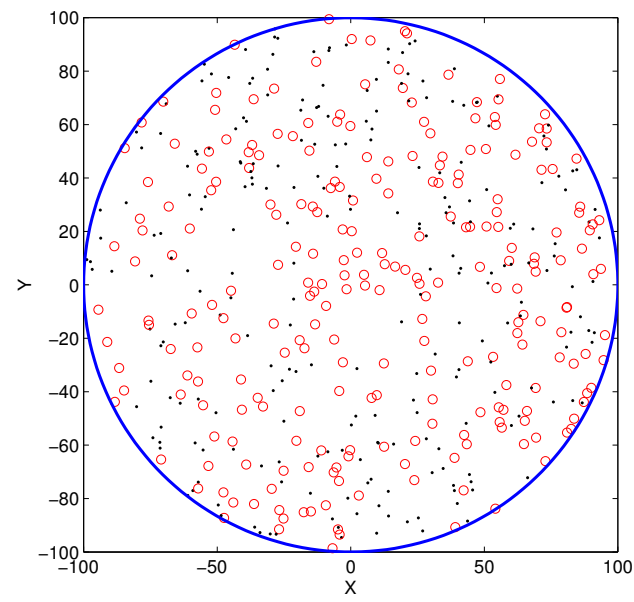

Fig. 14. Sensors that remain alive in the BS scheme after $50 \%$ of the sensor nodes run out of energy. Small circles represent alive sensor nodes, small dots represent dead sensor nodes. 
when the DS scheme is used, as shown in Fig. 13, the dead sensor nodes are still mostly in the cluster border. In contrast, when the BS scheme is used, as shown in Fig. 14, the distribution of alive and dead sensors is purely random. Therefore, the sensors that remain alive using the BS scheme will be better able to cover the entire cluster region than the sensors that remain alive using the RS or DS schemes.

\section{CONCLUSions}

In order to extend the network lifetime of wireless sensor networks, extra sensor nodes may be distributed to allow a certain fraction of the nodes to sleep from time to time. It is important to study the problem of how to select which sensors to put into the sleep mode in order to achieve maximum benefits from these sensor nodes, i.e., extending the network lifetime as much as possible while maintaining adequate sensing coverage.

In this paper, we studied the coefficient of variation of energy consumption of three different sleep scheduling schemes: the Randomized Scheduling (RS) scheme, the Distance-based Scheduling (DS) scheme, and the Balanced-energy Scheduling (BS) scheme. Our study shows that the proposed BS scheme extends the network lifetime by a factor of 1.5 and 0.7 compared with the RS and DS schemes, respectively.

In this work, we assumed that all sensors began with approximately the same amount of initial energy. In our future work, we will explore how the sleeping probabilities should change if nodes have different initial energy. In this case, the sleeping probabilities will need to be a function of $x$, the distance to the cluster head, as well as $E_{i}$, the energy of sensor $i$. In addition, we plan to investigate how cluster formation can benefit from these different sleep scheduling schemes, such as determining for a certain node distribution and sleep scheduling technique, the optimal number of clusters and the optimal cluster head locations. We will also explore ways to dynamically change clusters and cluster head nodes to ensure that all nodes are energy balanced while meeting the sensing requirements.

\section{ACKNOWLEDGMENT}

This work was supported in part by the SUPRIA program of the CASE Center at Syracuse University and by the National Science Council of Taiwan, R.O.C., under grants NSC 902213-E-260-007 and NSC 91-2213-E-260-021. We would also like to thank the anonymous reviewers for their valuable comments.

\section{REFERENCES}

[1] I. F. Akyildiz, W. Su, Y. Sankarasubramaniam, and E. Cayirci, "A survey on sensor networks," IEEE Communications Magazine, vol. 40, no. 8, pp. 102-114, August 2002.

[2] P. K. Varshney, Distributed Detection and Data Fusion, Springer, New York, 1997.

[3] W. R. Heinzelman, A. P. Chandrakasan, and H. Balakrishnan, "An application-specific protocol architecture for wireless microsensor networks," IEEE Transactions on Wireless Communications, vol. 1, no. 4, pp. 660-70, October 2002.

[4] Y. Xu, J. Heidemann, and D. Estrin, "Adaptive energy-conserving routing for multihop ad hoc networks," Research Report 527, USC/ISI, October 2000

[5] F. Stajano and R. Anderson, "The resurrecting duckling: Security issues in ad-hoc wireless networks," in Proc. of the 7th Int. Workshop on Security Protocols, Bruce Christianson, Bruno Crispo, and Mike Roe, Eds. 1999, Springer-Verlag.

[6] J. Deng, Y. S. Han, W. B. Heinzelman, and P. K. Varshney, "Scheduling sleeping nodes in high density cluster-based sensor networks," ACM/Kluwer Mobile Networks and Applications (MONET) Special Issue on "Energy Constraints and Lifetime Performance in Wireless Sensor Networks", 2004, to appear.

[7] M. Kochhal, L. Schwiebert, and S. Gupta, "Role-based hierarchical self-organization for wireless ad hoc sensor networks," in Proc. of the 2nd ACM Int. Workshop on Wireless Sensor Networks and Applications (WSNA '03). 2003, ACM Press.

[8] B. Chen, K. Jamieson, H. Balakrishnan, and R. Morris, "Span: an energy-efficient coordination algorithm for topology maintenance in ad hoc wireless networks," ACM Wireless Networks, vol. 8, no. 5, September 2002.

[9] D. Tian and N. D. Georganas, "A coverage-preserving node scheduling scheme for large wireless sensor networks," in Proc. of the 1st ACM Int. workshop on Wireless sensor networks and applications. 2002, pp. 32-41, ACM Press.

[10] W. Ye, J. Heidemann, and D. Estrin, "An energy-efficient MAC protocol for wireless sensor networks," in Proc. of the 21st Int. Annual Joint Conference of the IEEE Computer and Communications Societies (INFOCOM 2002), June 2002.

[11] M. R. Pearlman, J. Deng, B. Liang, and Z. J. Haas, "Elective participation in ad hoc networks based on energy consumption," in Proc. of IEEE Global Telecommunications Conference (GLOBECOM) / Symposium on Ad-Hoc Wireless Networks (SAWN), Taipei, Taiwan, November 17-21 2002, vol. 1, pp. 26-31.

[12] F. Ye, G. Zhong, S. Lu, and L. Zhang, "PEAS: A robust energy conserving protocol for long-lived sensor networks," in Proc. of the 23rd Int. Conf. on Distributed Computing Systems (ICDCS'03), May 2003.

[13] X. Wang, G. Xing, Y. Zhang, C. Lu, R. Pless, and C. D. Gill, "Integrated coverage and connectivity configuration in wireless sensor networks," in Proc. of the first Int. Conf. on Embedded networked sensor systems (SenSys'03), November 2003

[14] H. Zhang and J. C. Hou, "Maintaining sensing coverage and connectivity in large sensor networks," Technical Report 2351, UIUC, June 2003.

[15] Y. Yu and V. K. Prasanna, "Energy-balanced task allocation for collaborative processing in networked embedded systems," in Proc. of the 2003 ACM SIGPLAN conference on Language, compiler, and tool for embedded systems, 2003, pp. 265-274.

[16] J. Gomez and A. T. Campbell, "A case for variable-range transmission power control in wireless multihop networks," in Proc. of the 23rd Int. Annual Joint Conference of the IEEE Computer and Communications Societies (INFOCOM'04), March 2004.

[17] J. L. Gao, "Analysis of energy consumption for ad hoc wireless sensor networks using a bit-meter-per-joule metric," IPN Progress Report 42150, August 2002. 